\title{
edoc
}

Institutional Repository of the University of Basel

University Library

Schoenbeinstrasse 18-20

CH-4056 Basel, Switzerland

http://edoc.unibas.ch/

Year: 2012

\section{Bidirectional crosstalk between endoplasmic reticulum stress and mTOR signaling}

Appenzeller-Herzog, C. and Hall, M. N.

Posted at edoc, University of Basel

Official URL: http://edoc.unibas.ch/dok/A6002707

Originally published as:

Appenzeller-Herzog, C. and Hall, M. N.. (2012) Bidirectional crosstalk between endoplasmic reticulum stress and mTOR signaling. Trends in cell biology : TCB, Vol. 22, H. 5. S. 272-282.

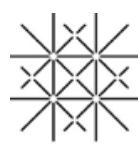


$8{ }^{1}$ Department of Pharmaceutical Sciences and ${ }^{2}$ Biozentrum, University of Basel,

9 Klingelbergstr. 50 / 70, CH-4056 Basel, Switzerland

*Corresponding authors:

14

15 Appenzeller-Herzog, C. (hristian.appenzeller@unibas.ch)

16 Hall, M. N. (m.hall@ unibas.ch)

17

18 Keywords: mTORC1, mTORC2, endoplasmic reticulum stress, unfolded protein 19 response, anabolism, apoptosis 


\section{Abstract}

23 Many cellular processes including apoptosis, autophagy, translation, energy

24 metabolism, and inflammation are controlled by the mammalian target of rapamycin

25 (mTOR) kinase and the endoplasmic reticulum (ER) stress pathway, also known as

26 the unfolded protein response (UPR). While both of these signaling nodes have

27 attracted wide attention in fundamental cell biology and drug discovery, crosstalk

28 between the two pathways has emerged only very recently. mTOR complex 1

29 (mTORC1) operates both upstream and downstream of ER stress signals, which can

30 either enhance or antagonize the anabolic output of mTORC1. Upon prolonged ER

31 stress, mTORC1 contributes to apoptotic signaling by suppressing the survival kinase

32 Akt through feedback inhibition. Likewise, chronic ER stress obstructs activation of

33 Akt by mTOR complex 2. This review surveys existing knowledge on mTOR-ER

34 stress intersections and highlights potential therapeutic implications. 


\section{Introduction}

38 Cell growth, proliferation, survival, and energetic maintenance are intimately 39 connected processes. External signals such as nutrient availability, growth factors, or 40 inflammatory mediators are decoded by cellular sentinels, which - where appropriate 41 - can remodel cell physiology. Thus, cells respond to positive growth signals by promoting anabolism (i.e. the buildup of macromolecules and the inhibition of degradative reactions) and to unfavorable growth conditions by eliciting stress pathways. The mammalian target of rapamycin (mTOR) signaling pathways and the endoplasmic reticulum (ER) stress response (the so-called "unfolded protein response", UPR) play increasingly recognized roles in this interplay [1, 2]. These two apposing signaling networks have traditionally been considered separate pathways and the identification of mTOR-UPR interconnections, which is reviewed herein, is a relatively new area of research.

50 The atypical serine/threonine kinase mTOR is a master regulator of cell growth and

51 metabolism. It exists in two complexes, mTORC1 and mTORC2, which exhibit 52 different subunit compositions and execute distinct cellular tasks [3, 4] (boxes 1 and 53 2). mTOR complexes reside in the cytoplasm, where they are often found in 54 association with cellular membranes ( see sections "Signal integration by mTORC1" 55 and "mTORC2 and the ER"). Several pathways downstream of the mTOR complexes 56 are known [4] and new ones are constantly being discovered. The UPR, on the other 57 hand, is a conglomeration of signaling pathways originating from the ER (box 3). It is 58 known to be triggered when the protein folding capacity in the ER is overwhelmed 59 and "ER stress" ensues. The membrane-bound network of the ER, which extends 60 from the nuclear envelope to the periphery of the cell and maintains vital contact 
61 zones with many other cell organelles, is a highly metabolic organelle [1]. It mediates many anabolic processes such as lipid synthesis, gluconeogenesis, and the biogenesis

63 of peroxisomes and lipid droplets, but also the catabolic turnover of proteins and 64 organelles through autophagy or proteasomal hydrolysis.

65 Signaling through mTORC1 and mTORC2 is activated by extracellular and 66 intracellular cues when conditions are favorable for growth. Both mTOR complexes 67 in turn facilitate cell growth, survival, and proliferation (boxes 1 and 2). The 68 metabolic classification of the UPR stress pathway is less straightforward, since it 69 would be a gross oversimplification to state that it generally antagonizes cellular 70 anabolism, which is orchestrated by mTOR. Indeed, the UPR does not only signal 71 stress, catabolism, and cell death [5], but also, for instance, the anabolic expansion of 72 ER membranes [6]. In the same vein, activation of the UPR is also achieved by 73 stimuli which are not necessarily linked to unfavorable ("stressful") growth 74 conditions (see section "Upstream of the UPR").

75 Given their central influence on cell viability, both mTOR and UPR have been subject 76 to extensive biomedical and pharmacological research activity [7-9], for instance in 77 the search for new cancer treatments. Thus, evaluating and understanding the 78 intersections and synergisms (or antagonisms) between the outputs of mTOR and 79 UPR is of importance; possible routes of crosstalk between these signaling networks 80 are fundamental for cell health. Here, we do not intend to provide a comprehensive 81 synopsis of the upstream and downstream signaling networks surrounding mTOR and 82 UPR for which the reader is referred to other recent articles [3-5, 10]. Instead, this 83 review focuses on the interdependence of these two pathways in health and disease. 84 Recent pioneering studies on molecular links between mTORC1, mTORC2, and the 
85 ER stress response will be summarized along with a tentative preview to where these new insights may guide future therapeutic strategies.

\section{Signal integration by mTORC1}

Our understanding of the molecular mechanisms leading to the activation of mTORC1 has tremendously increased over the past few years. Known pathways culminate in the association of mTORC1 with the active, GTP-bound forms of the small GTPases Rheb and Rag, which initiate mTORC1 signaling [11]. Thus, mTORC1 activation is regulated by at least two independent inputs. One is the regulation of the GTP-binding status of Rheb in response to growth factors. The other is the GTP loading of Rag and the recruitment of mTORC1 to the lysosomal membrane in response to amino acids $[4,11]$.

Growth factors and hormones, which operate upstream of Rheb-mTORC1, are recognized by cell surface receptors. These in turn initiate intracellular signaling cascades, the majority of which act on the heterodimeric TSC1-TSC2 (also called

101 hamartin-tuberin) complex, a GTPase activating complex and a negative regulator of

102 GTP-bound Rheb (Fig. 1). Examples of cell surface receptors which enhance mTORC1 signaling via inhibition of TSC1-TSC2 are the insulin receptor and

104 Frizzled (the receptor of the Wnt signaling pathway). The insulin receptor signals via 105 PI3K and PDK1 to the AGC kinase family member Akt. It also activates the MAP 106 kinase cascade Raf-Mek-Erk. Activated Akt and Erk phosphorylate and inactivate 107 TSC2 on multiple serine/threonine residues [12-14] (Fig. 1). In contrast, 108 phosphorylation by GSK3, which is inactivated by the Wnt-Frizzled pathway, 109 activates TSC2 [15]. Further regulatory inputs on TSC1-TSC2 by intracellular energy 
110 levels, cytokines, and hypoxia are summarized in Figure 1. Thus, the TSC1-TSC2

111 complex operates as an integrator of complex signaling information upstream of

112 mTORC1. Loss of either tumor suppressing subunit of TSC1-TSC2 leads to

113 constitutive activation of mTORC1 by Rheb-GTP and to an autosomal dominant

114 disease (tuberous sclerosis complex; TSC) characterized by the widespread

115 accumulation of benign tumors [16].

116 A second form of regulatory input on mTORC1 is spatial organization. Activation of

117 mTORC1 by Rheb-GTP occurs on the cytosolic surface of lysosomes. Recruitment of

118 mTORC1 to these sites is mediated by binding to heterodimeric Rag GTPases and

119 signaled by the availability of amino acids in lysosomes [17-19] (Fig. 1). In what way

120 this pathway is integrated with the input of alternative amino acid-sensing

121 machineries upstream of mTORC1 [20, 21] (Fig. 1) is currently unknown.

122 Collectively, activation of mTORC1 signaling is a multi-step process that depends on

123 the inputs of cellular signaling cascades and the availability of nutrients, energy, and

124 oxygen. As both Rheb- and Rag-integrated inputs are required for activation, this 125 creates a situation of tight regulation which - in light of the pathological 126 consequences of uncontrolled mTORC1 activation [16] - is critically important. 127 Before discussing the inputs of ER stress on mTORC1 activation, we will next give 128 an overview of specific "growth" conditions that elicit UPR signaling.

\section{Upstream of the UPR}

132 A growing body of evidence places the UPR downstream of physiological stimuli that 133 do not necessarily act via the accumulation of unfolded proteins in the ER [10]. For 134 instance, very much like mTORC1, the UPR is sensitive to the availability of 
nutrients and growth signals [1]. Indeed, the historically oldest way to elicit the UPR

136 (at that time measured by the increased synthesis of "glucose regulated proteins") was

137 glucose starvation [22], illustrating that the responsiveness of the ER to a low

138 nutritional or energetic state has been recognized for decades. UPR induction results,

139 at least in part, from a glucose-deprivation-induced decrease in cellular ATP, which

140 affects the function of the ER calcium pump SERCA2b and ER calcium levels [23].

141 Physiological UPR activation can therefore be mediated by unfavorable growth

142 conditions and be reciprocal to the activation of mTORC1.

143 Hypoxia represents another stress condition which activates UPR signaling and 144 inhibits mTORC1 [24]. What is the trigger of the UPR under hypoxic conditions?

145 One possible answer is given by the fact that Ero1 oxidases which support disulfide146 bond formation in nascent proteins in the ER depend on oxygen [25]. Accordingly, 147 lowered Ero1 activity under hypoxia could lead to hampered protein folding and ER 148 stress. This simple model, however, is complicated by the facts that oxygen 149 deprivation transcriptionally induces Ero1 $\alpha$ [26] and increases its activation state [27, 150 28]. Indeed, ER redox readouts showed that hypoxia-activated Ero1 $\alpha$ is operative in 151 preventing ER hypo-oxidation [27]. Alternative mechanisms are hypoxia-mediated 152 up-regulation of GSK3 $\beta$, which leads to the induction of PERK signaling via 153 destabilization of the nascent polypeptide-associated complex [29] and/or an increase 154 in free fatty acids (FFAs) evoked by the hypoxia-inducible transcription factor HIF$1552 \alpha[30]$.

156 The fact that circulating FFAs, prevalent during obesity, induce ER stress illustrates 157 that the UPR, like mTORC1, can also be activated by a high nutritional state [31]. 158 Underlying mechanisms likely include changes in ER membrane composition, 159 fluidity, and curvature that inhibit SERCA2b as shown in pancreatic $\beta$-cells [32] and 
mouse liver [33]. Other examples that document the responsiveness of the UPR to

161 overnutrition are cholesterol-loaded macrophages [34] and $\beta$-cells exposed to a high

162 glucose concentration [35].

163 In certain contexts, the UPR is also activated by growth stimuli. Thus, ER expansion

164 and induction of ER chaperones in B lymphocytes in response to antigen [36] and in 165 thyrocytes in response to thyroid-stimulating hormone [37] is mediated by the UPR.

166 Along the same line, UPR-mediated lipogenesis in the liver is activated following a 167 high-carbohydrate meal in an mTORC1-dependent manner [38]. This finding implies 168 that mTORC1 and UPR can act jointly to stimulate cell growth and suggests a 169 pathway by which mTORC1 can induce UPR signaling. Interestingly, the UPR170 dependent proliferation of ER membranes during differentiation of B lymphocytes 171 and thyrocytes precedes the massive synthesis of immunoglobulin and thyroglobulin, 172 respectively $[36,37]$, indicating that a mechanism other than ER overload is 173 responsible for UPR activation. Whether this mechanism involves mTORC1 has not 174 yet been examined.

175 Taken together, UPR signaling is elicited by a variety of physiological inputs, which 176 include both favorable and unfavorable growth conditions. Likewise, a functional 177 interaction between UPR and mTORC1 has been observed. As discussed in the next 178 section, bidirectional crosstalk between mTORC1 and UPR also occurs under 179 pathological conditions of chronic activation.

\section{Links between mTORC1 and UPR}

183 The primary output of UPR signaling is homeostatic adaptation by a variety of 184 mechanisms that aim at restoring ER function (box 3). As a secondary output, 
however, the UPR can also switch to promote apoptotic cell death through multiple pathways that remain to be fully understood [5]. Notably, the UPR as a mediator of ER-stress-induced apoptosis plays a pivotal role in a host of pathological conditions including neurodegenerative misfolding diseases and oxidative injury, as reviewed elsewhere $[9,39,40]$. It is intriguing to note that recent studies have also highlighted pathological situations where cell toxicity by ER stress is coupled to the chronic activation of mTORC1 [41-48]. This implies the apparent paradox that under certain settings, mTOR - a bona fide positive regulator of cell growth and division - can also signal cell demise. Furthermore, as discussed in this section, UPR activation can occur both upstream and downstream of mTORC1, which designates mTORC1 - at least in certain contexts - as a component in the process of ER-stress-induced cell death.

The best-documented ER pathway downstream of mTORC1 is Ire $1 \alpha-$ ASK1-JNK (box 3). Constitutive mTORC1 activation by loss of TSC1-TSC2 stimulates JNK, which contributes to ER-stress-induced apoptosis [41, 43, 48]. Furthermore, thus activated JNK can participate in the development of insulin resistance [43], which occurs in parallel to other mechanisms such as ER-stress-facilitated de novo lipogenesis [49], activation of PKR [50], and the mTORC1-S6K1-IRS1 negative feedback loop [51, 52]. Activation of the Ire1 $\alpha-\mathrm{JNK}$ branch downstream of mTORC1 appears to be privileged over other arms of the UPR [41], and it has been suggested that it is in fact the induction of an incomplete UPR by chronic mTORC1 activation that kills the cell [53]. Consistent with apoptotic signaling through mTORC1-Ire1 $\alpha-$ ASK1-JNK, over-expression or knockdown of Rheb enhances or antagonizes apoptotic stimuli in an ASK1-dependent fashion [54]. 
The finding that ER stress can also act upstream of mTORC1 adds a further layer of complexity. Pharmacological induction of the UPR rapidly activates the PI3K-Akt-

211 mTORC1 signaling axis [41, 44, 45], which depends on the ATF6 $\alpha$ branch of the 212 UPR [55]. Prolonged treatment with ER-stress-inducing agents, however, inhibits Akt $213[45,55-58]$ and mTORC1 $[42,59]$, which has - at least in part - been attributed to the 214 mTORC1-S6K1-IRS1 negative feedback loop [41, 52]. Furthermore, PERK-CHOP215 mediated induction of the Akt inhibitor TRB3 [60, 61] and GSK3 $\beta$-mediated 216 inactivation of mTORC2 (see section "mTORC2 and ER") may also contribute to the 217 inhibition of Akt upon advanced ER stress.

218 The suppression of Akt following an extended period of ER stress apparently plays a 219 central role in the activation of Ire1 $\alpha-$ ASK1-JNK downstream of mTORC1, possibly 220 by derepression of the ASK1 adaptor protein TRAF2 [41]. Such specific mTORC1221 to-ER signaling in our opinion better explains the selective activation of Ire $1 \alpha-J N K$ 222 upon chronic activation of mTORC1 than a general mTORC1-mediated increase in 223 protein synthesis and ER load. Additional modulation of UPR-mTORC1 crosstalk is 224 provided by ATF6 $\alpha$-dependent up-regulation of Rheb [62] and by Akt-catalyzed 225 suppressive phosphorylation of PERK [63]. The currently identified links between 226 mTORC1 and UPR both upon acute and chronic stimulation are depicted in Figure $2272 \mathrm{~A}$.

228 Given the interdependence of mTORC1 and UPR during pathological programs, it is 229 also interesting to consider common target processes as well as antagonizing outputs 230 of the two pathways during normal physiology. This interplay which is summarized in 231 Figure 2 is likely fundamental for cell health. Synergism between mTORC1 and UPR 232 occurs in the regulation of hepatic lipid synthesis [64-66], angiogenesis [67, 68], 233 NFkB signaling [1, 69], and insulin resistance [43, 51]. By contrast, the two pathways 
234 emit conflicting signals as to the control of ribosome biogenesis [70, 71], translation $235[4,10]$, apoptosis [5, 72], and autophagy [4, 73].

236 Collectively, convincing evidence exists that ER stress, through stimulation of the 237 "survival kinase" Akt, initially causes activation of mTORC1, which itself, in a later 238 phase, contributes to Akt inhibition to activate the ER-JNK "death kinase" pathway. 239 We therefore suggest that bi-phasic regulation of Akt by ER stress is a critical 240 determinant of apoptotic UPR signaling. As mTORC2 also participates in the

241 activation of Akt (box 2), the relationship between ER stress and mTORC2 is 242 discussed in the next section.

mTORC2 and the ER

246 In contrast to mTORC1, much less is known about pathways operating upstream of 247 mTORC2. However, ER stress also impacts mTORC2 signaling. Pharmacological 248 induction of ER stress for several hours leads to GSK3 $\beta$-catalyzed phosphorylation of 249 the mTORC2 component rictor, which suppresses Akt activation [74]. Thus, together 250 with the negative regulation of mTORC2 by the mTORC1 effector S6K1 [75], this 251 mechanism likely contributes to the chronic UPR-mTORC1 apoptosis pathway 252 described above.

253 Does mTORC2 also participate in Akt activation in an early phase of ER stress? At 254 present, there is no compelling evidence in favor or against this notion. However, as 255 demonstrated by immunofluorescence microscopy and subcellular fractionation, a 256 significant fraction of mTORC2 resides on ER membranes - even following the 257 stimulation of cells with growth factors [76]. It is therefore possible that 258 phosphorylation of Akt by mTORC2 occurs on the surface of the ER. Upstream 
regulation of mTORC2 critically relies on its binding to ribosomes, which is induced upon growth factor stimulation [77]. Whether such activation preferentially occurs on

261 ER-associated ribosomes and depends on the physiological state of the ER remains to 262 be explored. We would like to posit though that such regulation would be in line with 263 the emerging view that mTOR complexes are controlled by association with specific 264 membranes, as exemplified by mTORC1, which couples the sensing of amino acids in 265 the lysosomal lumen with its activation on the surface of lysosomes [19].

267 Therapeutic implications and future directions

The connections and interdependencies between mTORC1 and UPR during chronic responses are associated with various pathologies [43, 45-48]. As a consequence, a number of new clues for combined therapy ensue, which warrant detailed preclinical evaluations and are discussed in this section.

273 A first example is TSC [16], a multisystem disorder which is caused by constitutive 274 activation of mTORC1 and includes the activation of ER stress pathways [41, 43, 45, 275 53]. The most frequent medical symptom associated with TSC is epileptic seizure, 276 which is widely believed to be caused by cerebral cortical tubers [16] but has also 277 been proposed to be associated with mTORC1-dependent ER and oxidative stress 278 through the ATF4-CHOP pathway [45]. Accordingly, antioxidant therapy or 279 treatment with ER-stress-alleviating "chemical chaperones" such as 4-phenylbutyric 280 acid (PBA) possibly combined with a low dosage of an mTORC1 inhibitor such as 281 Everolimus (RAD001; approved for TSC patients who are not suitable for surgical 282 intervention [7]) might inhibit epileptic seizures in TSC. A concern with systemic 283 inhibition of mTORC1 in TSC, however, is that elimination of the S6K1-IRS1 
284 feedback loop would lead to hyper-activation of Akt, which could potentially convert 285 hamartomas into malignant tumors.

286 A deadly interplay between mTORC1 and ER stress has also been identified as a 287 causal factor in renal syndromes such as diabetic nephropathy $[47,78]$ and so-called 288 minimal change disease [46], which are characterized by the damage of glomerular 289 podocytes. Similarly, pancreatic $\beta$-cell demise under glucolipotoxic conditions that 290 occur in type 2 diabetes mellitus is supported by the same pathways [48]. In both 291 cases, however, the functioning of mTORC1 is essential for cell viability so that the 292 therapeutic value of mTOR inhibitors is restricted. It has therefore been suggested that 293 a low dosage of rapamycin or alternative mTORC1 inhibitor [7] that would lower but 294 not abolish mTORC1 signaling combined with PBA to inhibit ER stress could be used 295 for treatment of nephropathies [47]. A similar strategy could also be considered for 296 the treatment of type 2 diabetes, since combined inhibition of ER stress and mTORC1 297 is expected to improve the survival of $\beta$-cells [48] and also increase peripheral insulin 298 sensitivity [43]. Furthermore, mTORC1 inhibition could counteract the pathological 299 consequences of diabetes-linked obesity in white adipose tissue [79]. It is important to 300 emphasize though that despite the fact that both rapamycin and PBA are FDA301 approved drugs, such combined formulations for the treatment of TSC, renal failures, 302 or diabetes still require preclinical assessment to identify possible pleiotropic effects. 303 An alternative therapeutic approach to restore hepatic insulin sensitivity is the 304 stimulation of the deacetylase SIRT1, as adenovirus-mediated over-expression of 305 SIRT1 in the liver ameliorates the metabolic symptoms of obese animals through 306 inhibition of mTORC1 and ER stress [44]. In addition, clinically approved ASK1 or 307 JNK inhibitors would potentially add an important tool to mTORC1/ER-stress308 targeting combination therapies [9]. 
As exemplified in TSC cells which are particularly sensitive to ER-stress-induced cell death $[41,43,45,53]$ the targeted activation of this pathway could be used to

311 selectively kill tumors with hyperactive PI3K-Akt-mTORC1 axis. For instance, the 312 approved anti-tumor drug Velcade/Bortezomib which indirectly induces ER stress by 313 inhibiting the proteasome [80] could be considered. The Bcl-2 family tumor 314 suppressor Mcl-1 provides another promising link between mTORC1- and ER-stress315 based anti-tumor therapy. The inhibition of 4EBP-controlled translation of Mcl-1 is a 316 central element in the treatment of mTORC1-hyperactive cancers [72]. Since - with 317 the notable exception of melanoma cells [81] - the UPR also downregulates the 318 translation of this key antiapoptotic protein through PERK-eIF2 $\alpha$ [82, 83], a 319 combination of PERK induction [84] and mTOR inhibition [7] could produce 320 synergistic effects.

321 Although most of the links between mTOR and the ER have been uncovered by 322 examination of chronic responses, the two signaling nodes almost certainly also 323 interact under healthy conditions. Currently, this is best illustrated by the postprandial 324 up-regulation of Ire1 $\alpha$ signaling through mTORC1 in the liver [38]. As this up325 regulation is an acute anabolic response to mTORC1, it is reasonable to assume that it 326 does not result from inhibition of Akt, which elicits apoptotic Ire $1 \alpha$ signaling during 327 chronic response. A question for future investigation is, which factors make a cell 328 sensitive to the channeling of initially anabolic mTORC1 signals into the apoptotic 329 Ire1 $\alpha$-ASK1-JNK or ATF4-CHOP pathways. Moreover, it is important to stress that 330 the current mechanistic understanding of UPR-mTORC1 as well as of mTORC1331 UPR signaling is fragmentary (Fig. 2A). The linkage between UPR and the activation 332 of mTORC1 apparently involves the ATF6 $\alpha$ branch, which somehow elicits PI3K333 Akt signaling upstream of mTORC1 $[42,55]$ as well as the induction of Rheb [62]. 
334 The stimulation of Ire1 $\alpha$ by mTORC1, on the other hand, has been correlated with the 335 up-regulation of TRAF2 through depletion of activated Akt [41]. How Akt lowers the 336 levels of TRAF2, and whether there are additional mechanisms operating between 337 mTORC1 and the UPR machinery in parallel remains to be worked out.

338 In summary, a number of recent reports have shed light on new connections that link 339 two hitherto separated areas of modern cell biology. During the course of this review, 340 we have discussed both physiological and therapeutic implications of these findings. 341 Taken into consideration that the field of combined mTOR/UPR research is new, 342 significant progress is likely still ahead. 


\section{Box 1}

$345 \quad m$ TORC1

346 The catalytic subunit of mTORC1 (and mTORC2; box 2) is the PI3K-related protein

347 kinase family member mTOR, which associates with Raptor and mLST8 to form the

348 mTORC1 core complex [3] (Fig. I). Direct substrates of mTORC1 are 4E-BP1, S6K1,

349 ATG13, ULK1, and Lipin 1 [4, 8] (Fig. I). 4E-BP1 inhibits translation initiation

350 unless it is phosphorylated by mTORC1. Phosphorylated S6K1 positively regulates

351 mRNA translation and ribosome biosynthesis. Conversely, mTORC1 phosphorylates

352 and inhibits ATG13 and ULK1, thereby inhibiting autophagosome assembly. The

353 mechanism by which mTORC1 enhances lipid synthesis through SREBP transcription

354 factors has been elucidated only recently; Lipin 1, a negative regulator of SREBP

355 transcriptional activity, is prevented from entering the nucleus when phosphorylated

356 by mTORC1 [66]. Activation of mTORC1 also increases the transcription of HIF-1 $\alpha$

357 by an unknown mechanism, which stimulates glycolytic gene expression and 358 angiogenesis. 
Box 2

$361 \quad m T O R C 2$

362 The core components shared by mTORC2 and mTORC1 are mTOR and mLST8. In

363 addition, mTORC2 contains Rictor, mSIN1, which exists in several isoforms, and

364 PRR5 (also known as Protor1) (Fig. II). mTORC2 phosphorylates a subset of AGC

365 kinase family members on a conserved serine residue in the hydrophobic motif [4].

366 Identified substrates are the AGC kinases Akt, SGK1, and PKC (Fig. II). Ablation of 367 mTORC2 does not equally affect all Akt substrates; for example, phosphorylation of 368 the FOXO1 and 3 transcription factors is affected, whereas phosphorylation of TSC2, 369 which is upstream of mTORC1, is not affected [85]. By phosphorylating PKC, 370 mTORC2 also regulates the actin cytoskeleton and cell polarity. 


\section{Box 3}

373 The Unfolded Protein Response

374 UPR signaling in vertebrates depends on three types of ER membrane-embedded sensor proteins that impart distinct, but partially overlapping cell fate signals $[1,10]$ (Fig. III). (i) After UPR activation, the Ire1 $\alpha$ RNase initiates the unconventional 377 splicing of a specific mRNA on the surface of the ER, which then encodes the transcription factor Xbp1s. Alternatively, probably depending on the nature or severity of the triggering insult, Ire $1 \alpha$ can both, degrade select ER-associated mRNAs

380 (through a process called regulated Ire1 $\alpha$-dependent decay; RIDD) to attenuate 381 protein import into the ER and initiate a MAP kinase signaling cascade that leads to JNK activation. (ii) By directly phosphorylating the eIF2 $\alpha$ translation initiation factor, the ER stress-sensor PERK is part of an "integrated stress response", which lowers overall translation, while increasing the cellular antioxidant capacity by selectively stimulating the translation of the transcription factor ATF4 among others. In addition, PERK directly activates the antioxidant response transcription factor Nrf2. (iii) The 387 activation of ATF6 $\alpha$ and an increasing number of tissue-specific ATF6-like ERresident transcription factors occurs by yet another mechanism; on sensing ER stress, these proteins travel to the Golgi complex where they are subjected to intramembrane 390 proteolysis thus liberating their DNA-binding domain (p50) for nuclear translocation. 391 Transcriptional targets of Xbp1s, ATF4, and ATF6 $\alpha$ include genes encoding ER 392 chaperones and oxidoreductases, ER-associated degradation factors, phospholipid 393 biosynthesis enzymes, and proteins involved in metabolic control.

394 While the UPR primarily aims at lowering the burden of folding substrates and 395 increasing the capacity of the ER's folding machinery, it can also mediate apoptotic 396 cell death through many different pathways [5]. One key pathway to ER stress- 
397 induced apoptosis is the transcriptional induction of CHOP by ATF4 (Fig. III). CHOP 398 is an important proapoptotic transcription factor that, for instance, upregulates 399 proapoptotic members of the Bcl-2 family. Likewise, the activation of the intrinsic 400 apoptosis pathway via ER stress-induced ER-to-mitochondria calcium transmission 401 and activation of Bax/Bak on mitochondria is of particular importance [5].

402 
Figure legends

Figure 1

\section{Upstream regulation of $\mathrm{mTORC1}$}

Activation of mTORC1 is controlled by TSC1-TSC2 and by amino acids (AA).

(Upper part) By acting as a GTPase-activating protein on Rheb, TSC1-TSC2 inhibits mTORC1 signaling. TSC1-TSC2 loss-of-function systems are frequently used to study the consequences of chronic mTORC1 hyper-activity. Multiple pathways

411 positively or negatively impact mTORC1 signaling through modulation of TSC1-

412 TSC2. Growth factors and hormones signal via PI3K-Akt or Raf-Mek-Erk to 413 phosphorylate and inhibit TSC1-TSC2. Conversely, GSK3 activates TSC2 by 414 phosphorylation, as does AMPK in response to low intracellular energy levels [86]. 415 Similarly, a cytokine-induced pathway where IKK $\beta$ phosphorylates and suppresses 416 TSC1 exists to varying degrees in different cell types [87]. In addition to these 417 phosphorylation-dependent events, the stability of TSC1-TSC2 is negatively 418 regulated through association with the gluconeogenic (i.e. anabolic) transcription 419 factor FOXO1 [88]. Furthermore, an inhibitory complex between TSC2 and 14-3-3 420 proteins is dissociated by the action of REDD1 in response to hypoxia [89], which 421 adds to several different mechanisms for mTORC1 inhibition by hypoxia [24]. Where 422 applicable, the principal phosphorylation sites of a given pathway in TSC1-TSC2, 423 which either enhance or suppress its activity, are indicated. (Lower part) Different 424 mechanisms by which AA talk to mTORC1 exist in parallel. (1) In mammals, AA 425 stimulation induces influx of extracellular $\mathrm{Ca}^{2+}$, which activates calmodulin $(\mathrm{CaM})$ to 426 bind and activate the class III PI3K mVps34. This complex in turn positively 427 regulates mTORC1 by a mechanism that remains to be fully elucidated [21]. (2) AA 
428 in the lysosomal lumen modulate the binding of the vacuolar proton pump (v429 ATPase) to the trimeric p14-p18-MP1 complex (also called the Ragulator), which 430 anchors Rag GTPases to the surface of lysosomes. Thus, v-ATPase promotes the

431 translocation of mTORC1 to lysosomes where it is activated by Rheb-GTP [19]. (3)

432 Phosphorylation of MAP4K3 on Ser170 in response to AA activates mTORC1 by an 433 unknown mechanism [20].

Figure 2

\section{Interplay between UPR and mTORC1}

437 (A) The known signaling pathways linking UPR and mTORC1 activation can be 438 subdivided into acute phase (black arrows) and chronic phase (grey arrows) pathways. 439 ER stress/UPR can activate mTORC1 via ATF6 $\alpha$, which triggers the PI3K pathway 440 and increases the levels of Rheb by unknown mechanisms (question marks). The 441 former pathway leads to activation of Akt during an early phase of ER stress. Acute 442 activation of the UPR by mTORC1 presumably occurs through increased protein 443 synthesis, which elevates the demand on the ER machinery for protein folding. 444 Chronic activation of mTORC1 and UPR causes inactivation of Akt through at least 445 four mechanisms: (1) Suppressive phosphorylation of IRS1 and (2) mTORC2 by 446 S6K1 downstream of mTORC1, (3) inhibition of mTORC2 by GSK3ß-catalyzed 447 phosphorylation downstream of ER stress, and (4) PERK-CHOP-mediated induction 448 of TRB3, which directly binds to Akt and blocks its activation. Lowered Akt 449 activation precipitates higher levels of TRAF2, which triggers the Ire1 $\alpha-$ ASK1-JNK 450 branch of the UPR. Question marks denote where molecular mechanisms remain to be 451 identified. 
452 (B) The diverse outputs of the UPR and mTORC1 pathways can be synergistic or 453 antagonistic. Joint positive regulation occurs in the case of de novo lipogenesis, 454 angiogenesis, insulin resistance, and activation of the NFkB pathway (light brown 455 boxes). By contrast, autophagy is stimulated by the UPR and inhibited by mTORC1, 456 whereas inverse signaling outputs regulate the processes of ribosome biogenesis and 457 translation (light green boxes). Relating to apoptosis, the interplay between UPR and 458 mTORC1 is context-dependent. While in an initial ("physiological") phase of ER 459 stress, the output of the UPR may be homeostatic/antiapoptotic, the UPR will promote 460 apoptosis upon chronic activation (represented by a dashed activation arrow). 461 Likewise, on unknown stimulus and/or prolonged ER stress, mTORC1, which usually 462 promotes cell survival, can also contribute to apoptotic signaling by the UPR (dashed 463 grey arrow; see main text for details). 


\section{Acknowledgements}

466 We thank Don Benjamin for critical review of the manuscript. Funding by the Canton

467 of Basel, the Swiss National Science Foundation (CAH and MNH), the August 468 Collin-Fonds (CAH), and the Louis-Jeantet Foundation (MNH) is gratefully 469 acknowledged.

470 


\section{References}

472

473

474

475

476

477

478

479

480

481

482

483

484

485

486

487

488

489

490

491

492

493

494

495

496

497

498

499

500

501

502

503

504

505

506

507

508

509

510

511

512

513

514

515

516

517

518

1 Hotamisligil, G.S. (2010) Endoplasmic reticulum stress and the inflammatory basis of metabolic disease. Cell 140, 900-917

2 Polak, P., and Hall, M.N. (2009) mTOR and the control of whole body metabolism. Curr Opin Cell Biol 21, 209-218

3 Wullschleger, S., et al. (2006) TOR signaling in growth and metabolism. Cell 124, 471-484

4 Zoncu, R., et al. (2011) mTOR: from growth signal integration to cancer, diabetes and ageing. Nat Rev Mol Cell Biol 12, 21-35

5 Tabas, I., and Ron, D. (2011) Integrating the mechanisms of apoptosis induced by endoplasmic reticulum stress. Nat Cell Biol 13, 184-190

6 Schuck, S., et al. (2009) Membrane expansion alleviates endoplasmic reticulum stress independently of the unfolded protein response. J Cell Biol 187, 525-536

7 Benjamin, D., et al. (2011) Rapamycin passes the torch: a new generation of mTOR inhibitors. Nat Rev Drug Discov 10, 868-880

8 Dazert, E., and Hall, M.N. (2011) mTOR signaling in disease. Curr Opin Cell Biol 23, 744-755

9 Kim, I., et al. (2008) Cell death and endoplasmic reticulum stress: disease relevance and therapeutic opportunities. Nat Rev Drug Discov 7, 1013-1030

10 Rutkowski, D.T., and Hegde, R.S. (2010) Regulation of basal cellular physiology by the homeostatic unfolded protein response. J Cell Biol 189, 783-794

11 Duran, R.V., and Hall, M.N. (2012) Regulation of TOR by small GTPases. EMBO Rep in press

12 Inoki, K., et al. (2002) TSC2 is phosphorylated and inhibited by Akt and suppresses mTOR signalling. Nat Cell Biol 4, 648-657

13 Ma, L., et al. (2005) Phosphorylation and functional inactivation of TSC2 by Erk implications for tuberous sclerosis and cancer pathogenesis. Cell 121, 179-193

14 Manning, B.D., et al. (2002) Identification of the tuberous sclerosis complex-2 tumor suppressor gene product tuberin as a target of the phosphoinositide 3kinase/akt pathway. Mol Cell 10, 151-162

15 Inoki, K., et al. (2006) TSC2 integrates Wnt and energy signals via a coordinated phosphorylation by AMPK and GSK3 to regulate cell growth. Cell 126, 955-968

16 Crino, P.B., et al. (2006) The tuberous sclerosis complex. $N$ Engl J Med 355, $1345-1356$

17 Sancak, Y., et al. (2008) The Rag GTPases bind raptor and mediate amino acid signaling to mTORC1. Science 320, 1496-1501

$18 \mathrm{Kim}$, E., et al. (2008) Regulation of TORC1 by Rag GTPases in nutrient response. Nat Cell Biol 10, 935-945

19 Zoncu, R., et al. (2011) mTORC1 senses lysosomal amino acids through an inside-out mechanism that requires the vacuolar H-ATPase. Science 334, 678-683

20 Yan, L., et al. (2010) PP2A T61 epsilon is an inhibitor of MAP4K3 in nutrient signaling to mTOR. Mol Cell 37, 633-642

21 Gulati, P., et al. (2008) Amino acids activate mTOR complex 1 via $\mathrm{Ca} 2+/ \mathrm{CaM}$ signaling to hVps34. Cell Metab 7, 456-465

22 Lee, A.S. (1987) Coordinated regulation of a set of genes by glucose and calcium ionophores in mammalian cells. Trends Biochem Sci 12, 20-23 
23 Moore, C.E., et al. (2011) PERK activation at low glucose concentration is mediated by SERCA pump inhibition and confers preemptive cytoprotection to pancreatic beta-cells. Mol Endocrinol 25, 315-326

24 Wouters, B.G., and Koritzinsky, M. (2008) Hypoxia signalling through mTOR and the unfolded protein response in cancer. Nat Rev Cancer 8, 851-864

25 Ramming, T., and Appenzeller-Herzog, C. (2012) The physiological functions of mammalian endoplasmic oxidoreductin 1 (Ero1): on disulfides and more. Antioxid Redox Signal in press

26 May, D., et al. (2005) Ero1-L alpha plays a key role in a HIF-1-mediated pathway to improve disulfide bond formation and VEGF secretion under hypoxia: implication for cancer. Oncogene 24, 1011-1020

27 Chin, K.T., et al. (2011) The sarcoplasmic reticulum luminal thiol oxidase ERO1 regulates cardiomyocyte excitation-coupled calcium release and response to hemodynamic load. FASEB $J$ 25, 2583-2591

28 Appenzeller-Herzog, C., et al. (2008) A novel disulphide switch mechanism in Erolalpha balances ER oxidation in human cells. EMBO J 27, 2977-2987

29 Hotokezaka, Y., et al. (2009) alphaNAC depletion as an initiator of ER stressinduced apoptosis in hypoxia. Cell Death Differ 16, 1505-1514

$30 \mathrm{Qu}$, A., et al. (2011) Hypoxia-inducible transcription factor 2alpha promotes steatohepatitis through augmenting lipid accumulation, inflammation, and fibrosis. Hepatology 54, 472-483

31 Pineau, L., and Ferreira, T. (2010) Lipid-induced ER stress in yeast and beta cells: parallel trails to a common fate. FEMS Yeast Res 10, 1035-1045

32 Cunha, D.A., et al. (2008) Initiation and execution of lipotoxic ER stress in pancreatic beta-cells. J Cell Sci 121, 2308-2318

$33 \mathrm{Fu}$, S., et al. (2011) Aberrant lipid metabolism disrupts calcium homeostasis causing liver endoplasmic reticulum stress in obesity. Nature 473, 528-531

34 Tabas, I. (2010) The role of endoplasmic reticulum stress in the progression of atherosclerosis. Circ Res 107, 839-850

35 Leibowitz, G., et al. (2010) Glucose regulation of beta-cell stress in type 2 diabetes. Diabetes Obes Metab 12 Suppl 2, 66-75

36 van Anken, E., et al. (2003) Sequential waves of functionally related proteins are expressed when B cells prepare for antibody secretion. Immunity 18, 243-253

37 Christis, C., et al. (2010) Regulated increase in folding capacity prevents unfolded protein stress in the ER. J Cell Sci 123, 787-794

38 Pfaffenbach, K.T., et al. (2010) Rapamycin inhibits postprandial-mediated X-boxbinding protein-1 splicing in rat liver. $J$ Nutr 140, 879-884

39 Matus, S., et al. (2011) Protein folding stress in neurodegenerative diseases: a glimpse into the ER. Curr Opin Cell Biol 23, 239-252

40 Santos, C.X., et al. (2009) Mechanisms and implications of reactive oxygen species generation during the unfolded protein response: roles of endoplasmic reticulum oxidoreductases, mitochondrial electron transport, and NADPH oxidase. Antioxid Redox Signal 11, 2409-2427

41 Kato, H., et al. (2011) mTORC1 serves ER stress-triggered apoptosis via selective activation of the IRE1-JNK pathway. Cell Death Differ

42 Nakajima, S., et al. (2011) Selective abrogation of BiP/GRP78 blunts activation of NF-kappaB through the ATF6 branch of the UPR: involvement of C/EBPbeta and mTOR-dependent dephosphorylation of Akt. Mol Cell Biol 31, 1710-1718 
43 Ozcan, U., et al. (2008) Loss of the tuberous sclerosis complex tumor suppressors triggers the unfolded protein response to regulate insulin signaling and apoptosis. Mol Cell 29, 541-551

$44 \mathrm{Li}$, Y., et al. (2011) Hepatic overexpression of SIRT1 in mice attenuates endoplasmic reticulum stress and insulin resistance in the liver. FASEB $J 25$, 1664-1679

45 Di Nardo, A., et al. (2009) Tuberous sclerosis complex activity is required to control neuronal stress responses in an mTOR-dependent manner. J Neurosci 29, 5926-5937

46 Ito, N., et al. (2011) mTORC1 activation triggers the unfolded protein response in podocytes and leads to nephrotic syndrome. Lab Invest 91, 1584-1595

47 Inoki, K., et al. (2011) mTORC1 activation in podocytes is a critical step in the development of diabetic nephropathy in mice. J Clin Invest 121, 2181-2196

48 Bachar, E., et al. (2009) Glucose amplifies fatty acid-induced endoplasmic reticulum stress in pancreatic beta-cells via activation of mTORC1. PLoS One 4, e4954

49 Jurczak, M.J., et al. (2012) Dissociation of inositol requiring enzyme (IRE1alpha)-mediated JNK activation from hepatic insulin resistance in conditional X-box binding protein-1 (XBP1) knockout mice. J Biol Chem

50 Nakamura, T., et al. (2010) Double-stranded RNA-dependent protein kinase links pathogen sensing with stress and metabolic homeostasis. Cell 140, 338-348

$51 \mathrm{Um}, \mathrm{S}$.H., et al. (2004) Absence of S6K1 protects against age- and diet-induced obesity while enhancing insulin sensitivity. Nature 431, 200-205

52 Tremblay, F., et al. (2007) Identification of IRS-1 Ser-1101 as a target of S6K1 in nutrient- and obesity-induced insulin resistance. Proc Natl Acad Sci U S A 104, 14056-14061

53 Kang, Y.J., et al. (2010) The TSC1 and TSC2 tumor suppressors are required for proper ER stress response and protect cells from ER stress-induced apoptosis. Cell Death Differ 18, 133-144

54 Karassek, S., et al. (2010) Ras homolog enriched in brain (Rheb) enhances apoptotic signaling. J Biol Chem 285, 33979-33991

55 Yamazaki, H., et al. (2009) Activation of the Akt-NF-kappaB pathway by subtilase cytotoxin through the ATF6 branch of the unfolded protein response. $J$ Immunol 183, 1480-1487

56 Ishigaki, S., et al. (2010) AATF mediates an antiapoptotic effect of the unfolded protein response through transcriptional regulation of AKT1. Cell Death Differ 17, 774-786

$57 \mathrm{Hu}$, P., et al. (2004) Critical role of endogenous Akt/IAPs and MEK1/ERK pathways in counteracting endoplasmic reticulum stress-induced cell death. $J$ Biol Chem 279, 49420-49429

58 Hosoi, T., et al. (2007) Akt up- and down-regulation in response to endoplasmic reticulum stress. Brain Res 1152, 27-31

59 Deldicque, L., et al. (2011) ER stress induces anabolic resistance in muscle cells through PKB-induced blockade of mTORC1. PLoS One 6, e20993

60 Du, K., et al. (2003) TRB3: a tribbles homolog that inhibits Akt/PKB activation by insulin in liver. Science 300, 1574-1577

61 Ohoka, N., et al. (2005) TRB3, a novel ER stress-inducible gene, is induced via ATF4-CHOP pathway and is involved in cell death. EMBO J 24, 1243-1255 
62 Schewe, D.M., and Aguirre-Ghiso, J.A. (2008) ATF6alpha-Rheb-mTOR signaling promotes survival of dormant tumor cells in vivo. Proc Natl Acad Sci U S A 105, 10519-10524

63 Mounir, Z., et al. (2011) Akt determines cell fate through inhibition of the PERKeIF2alpha phosphorylation pathway. Sci Signal 4, ra62

64 Lee, A.H., et al. (2008) Regulation of hepatic lipogenesis by the transcription factor XBP1. Science 320, 1492-1496

65 Kammoun, H.L., et al. (2009) GRP78 expression inhibits insulin and ER stressinduced SREBP-1c activation and reduces hepatic steatosis in mice. J Clin Invest $119,1201-1215$

66 Peterson, T.R., et al. (2011) mTOR complex 1 regulates lipin 1 localization to control the SREBP pathway. Cell 146, 408-420

67 Thomas, G.V., et al. (2006) Hypoxia-inducible factor determines sensitivity to inhibitors of mTOR in kidney cancer. Nat Med 12, 122-127

68 Pereira, E.R., et al. (2010) Transcriptional and post-transcriptional regulation of proangiogenic factors by the unfolded protein response. PLoS One 5

69 Dan, H.C., et al. (2008) Akt-dependent regulation of NF-\{kappa $\}$ B is controlled by mTOR and Raptor in association with IKK. Genes Dev 22, 1490-1500

70 Mayer, C., et al. (2004) mTOR-dependent activation of the transcription factor TIF-IA links rRNA synthesis to nutrient availability. Genes Dev 18, 423-434

71 DuRose, J.B., et al. (2009) Phosphorylation of eukaryotic translation initiation factor 2alpha coordinates rRNA transcription and translation inhibition during endoplasmic reticulum stress. Mol Cell Biol 29, 4295-4307

72 Hsieh, A.C., et al. (2010) Genetic dissection of the oncogenic mTOR pathway reveals druggable addiction to translational control via 4EBP-eIF4E. Cancer Cell 17, 249-261

73 Hoyer-Hansen, M., and Jaattela, M. (2007) Connecting endoplasmic reticulum stress to autophagy by unfolded protein response and calcium. Cell Death Differ 14, 1576-1582

74 Chen, C.H., et al. (2011) ER stress inhibits mTORC2 and Akt signaling through GSK-3beta-mediated phosphorylation of rictor. Sci Signal 4, ra10

75 Dibble, C.C., et al. (2009) Characterization of Rictor phosphorylation sites reveals direct regulation of mTOR complex 2 by S6K1. Mol Cell Biol 29, 5657-5670

76 Boulbes, D.R., et al. (2011) Endoplasmic reticulum is a main localization site of mTORC2. Biochem Biophys Res Commun 413, 46-52

77 Zinzalla, V., et al. (2011) Activation of mTORC2 by association with the ribosome. Cell 144, 757-768

78 Godel, M., et al. (2011) Role of mTOR in podocyte function and diabetic nephropathy in humans and mice. J Clin Invest 121, 2197-2209

79 Polak, P., et al. (2008) Adipose-specific knockout of raptor results in lean mice with enhanced mitochondrial respiration. Cell Metab 8, 399-410

80 Lee, A.H., et al. (2003) Proteasome inhibitors disrupt the unfolded protein response in myeloma cells. Proc Natl Acad Sci U S A 100, 9946-9951

81 Dong, L., et al. (2011) Ets-1 mediates upregulation of Mcl-1 downstream of XBP1 in human melanoma cells upon ER stress. Oncogene 30, 3716-3726

82 Morishima, N., et al. (2011) Activating transcription factor-6 (ATF6) mediates apoptosis with reduction of myeloid cell leukemia sequence $1(\mathrm{Mcl}-1)$ protein via induction of WW domain binding protein 1. J Biol Chem 286, 35227-35235 
663

664

665

666

667

668

669

670

671

672

673

674

675

676

677

678

679

680

681

682

683

83 Allagnat, F., et al. (2011) Mcl-1 downregulation by pro-inflammatory cytokines and palmitate is an early event contributing to beta-cell apoptosis. Cell Death Differ 18, 328-337

84 Stockwell, S.R., et al. (2012) Mechanism-Based Screen for G1/S Checkpoint Activators Identifies a Selective Activator of EIF2AK3/PERK Signalling. PLoS One 7, e28568

85 Polak, P., and Hall, M.N. (2006) mTORC2 Caught in a SINful Akt. Dev Cell 11, 433-434

86 Inoki, K., et al. (2003) TSC2 mediates cellular energy response to control cell growth and survival. Cell 115, 577-590

87 Dan, H.C., and Baldwin, A.S. (2008) Differential involvement of IkappaB kinases alpha and beta in cytokine- and insulin-induced mammalian target of rapamycin activation determined by Akt. J Immunol 180, $7582-7589$

88 Cao, Y., et al. (2006) Interaction of FoxO1 and TSC2 induces insulin resistance through activation of the mammalian target of rapamycin/p70 S6K pathway. $J$ Biol Chem 281, 40242-40251

89 DeYoung, M.P., et al. (2008) Hypoxia regulates TSC1/2-mTOR signaling and tumor suppression through REDD1-mediated 14-3-3 shuttling. Genes Dev 22, 239-251 

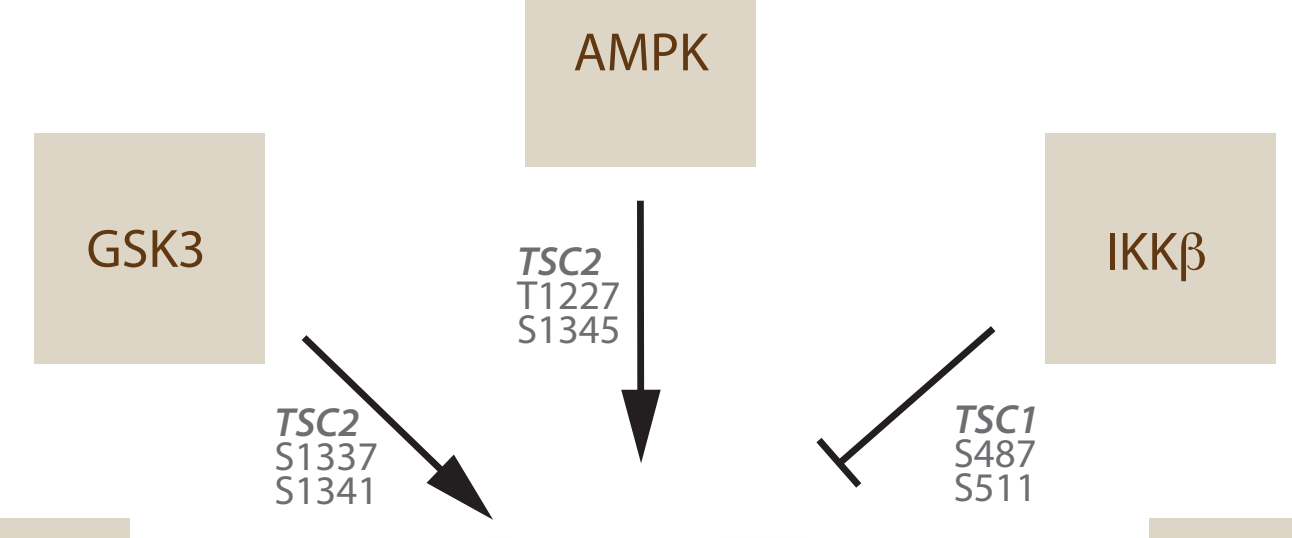

Raf Mek Erk
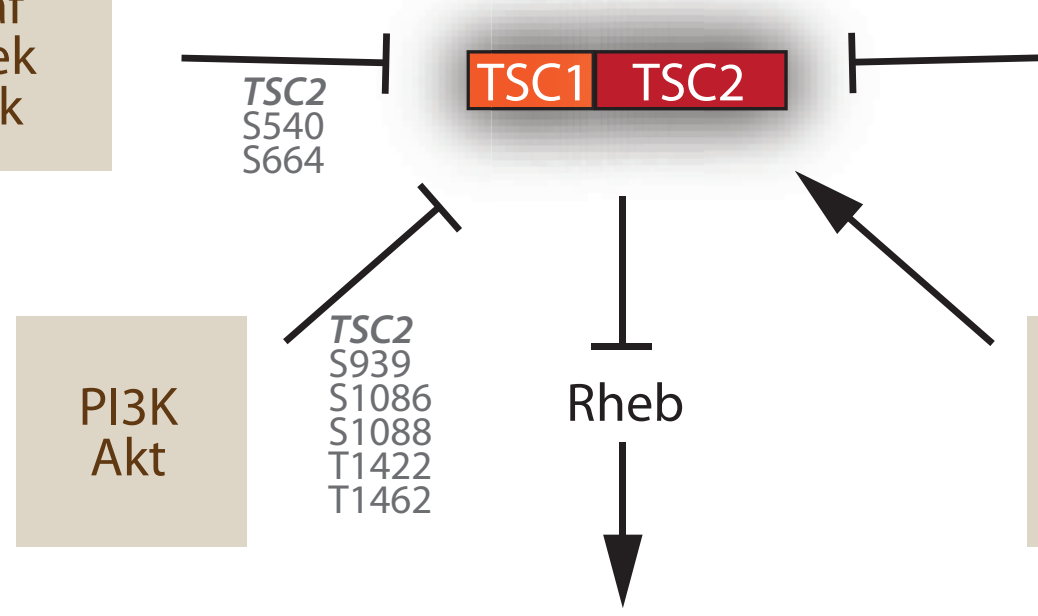

FOXO1

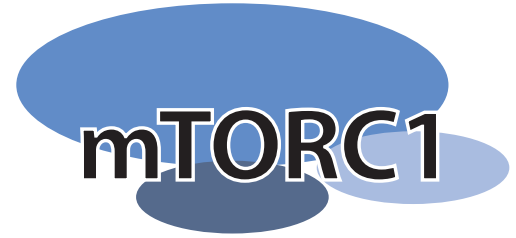

(1)

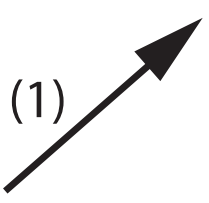

mVps34

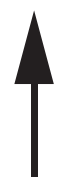

$\mathrm{Ca}^{2+}-\mathrm{CaM}$

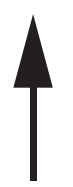

AA

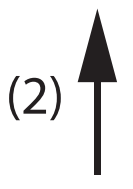

RagA/B-RagC/D

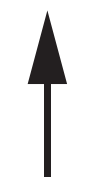

p14 MP1

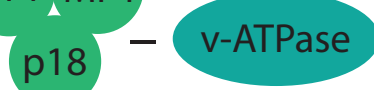

4

AA (lysosomal)

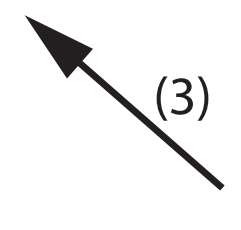

MAP4K3

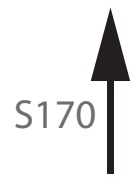

AA 


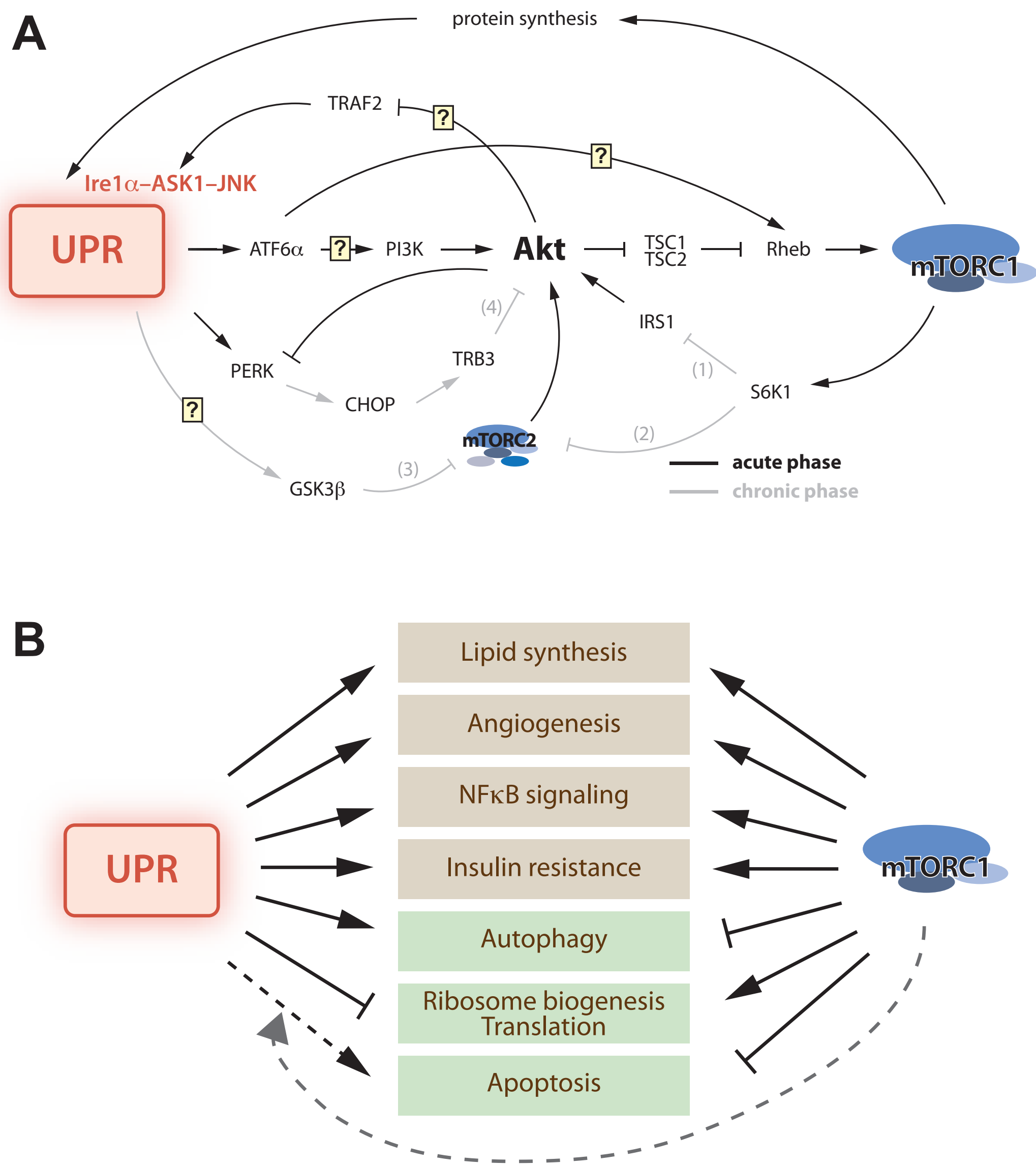




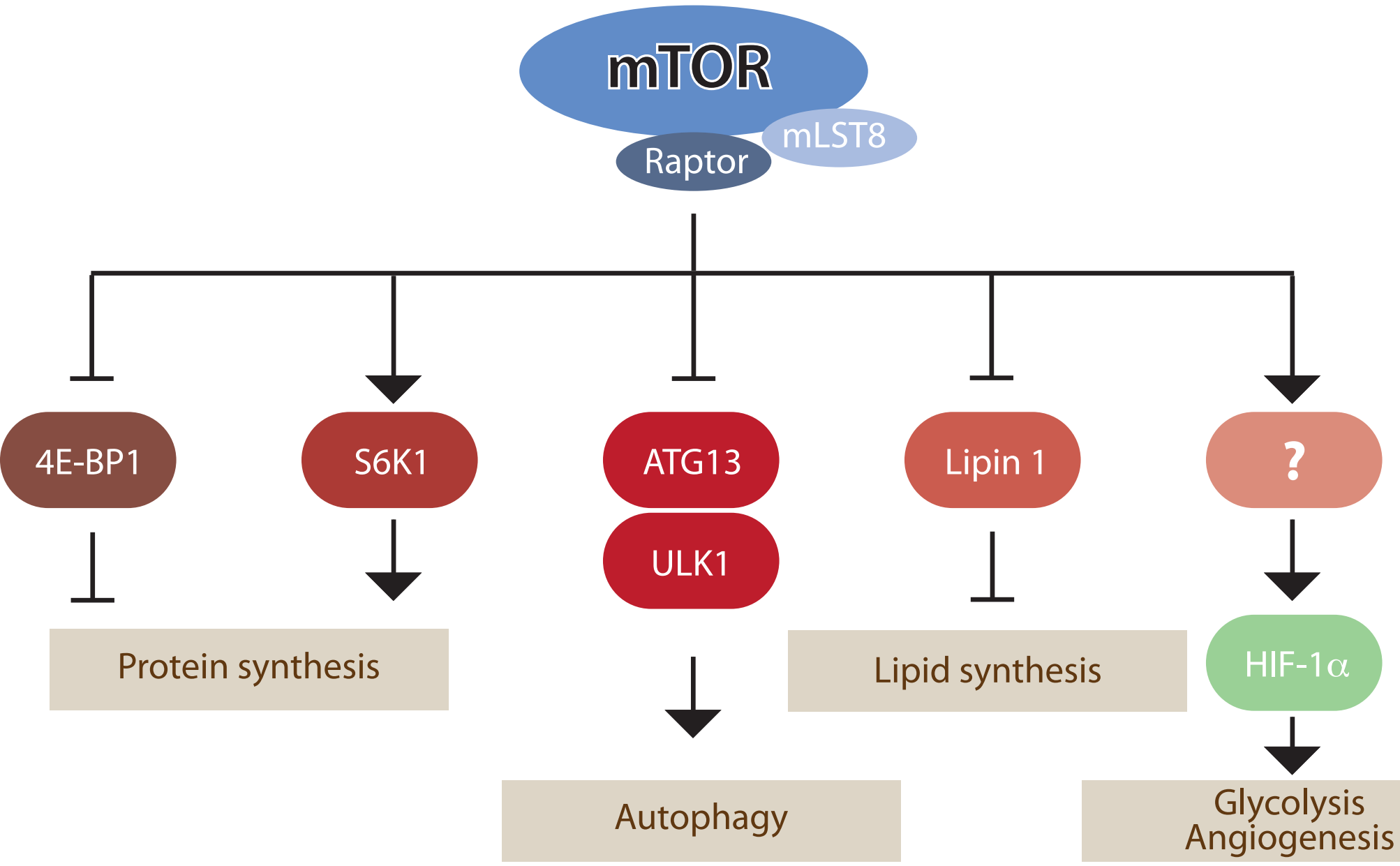

Appenzeller-Herzog Figure I

\section{ULK1} Angiogenesis

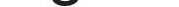



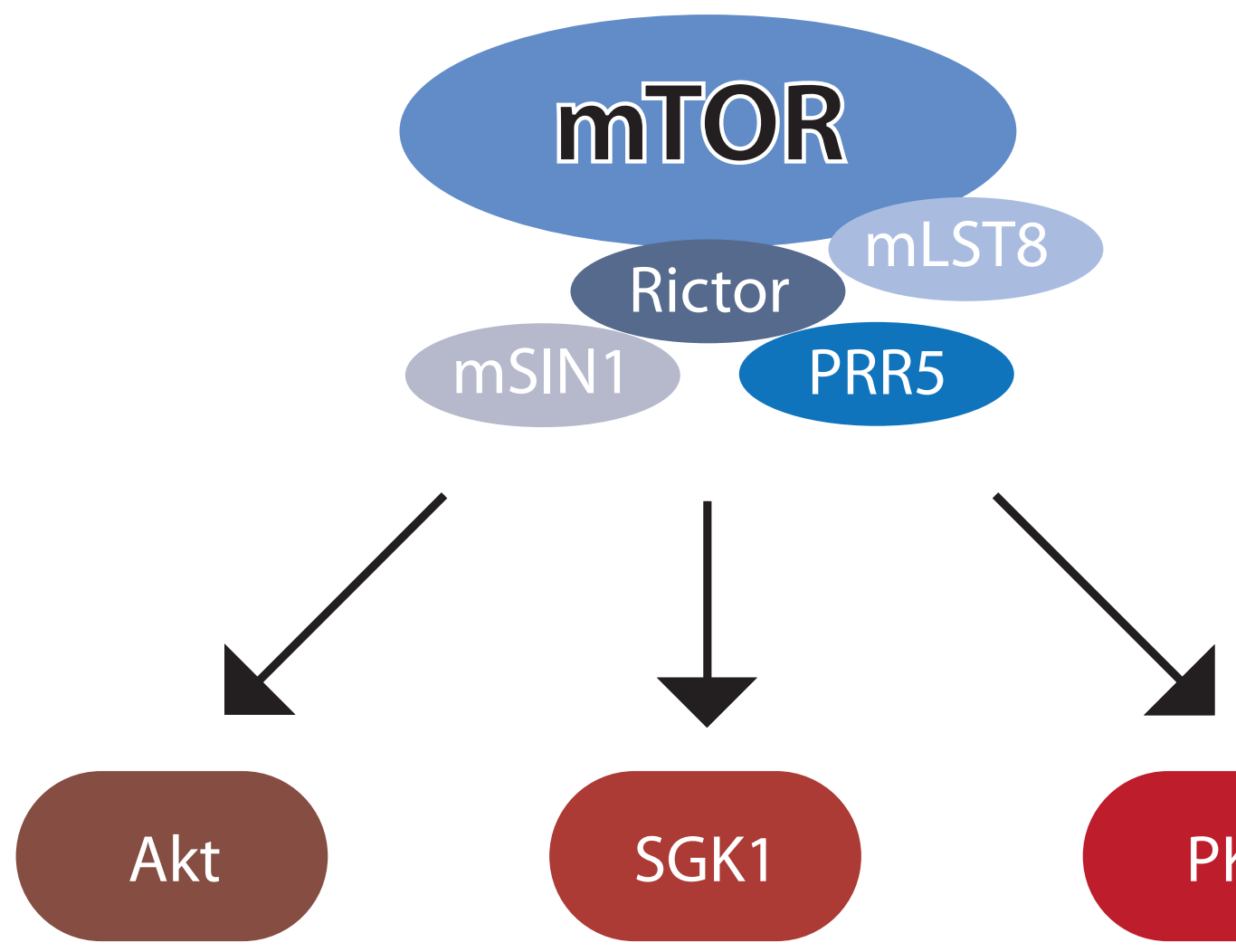

\section{SGK1}

PKC

1

\section{$\mathrm{FOX01/3}$}

Cytoskeletal organisation
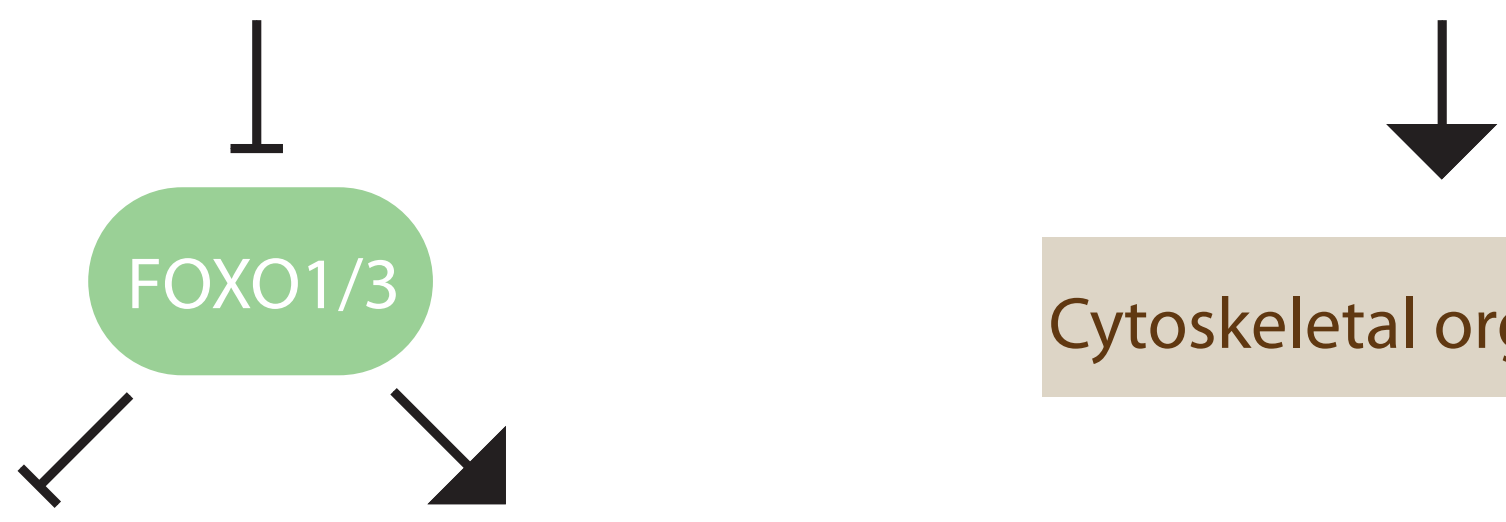

Cell survival Gluconeogenesis

Appenzeller-Herzog Figure II 
